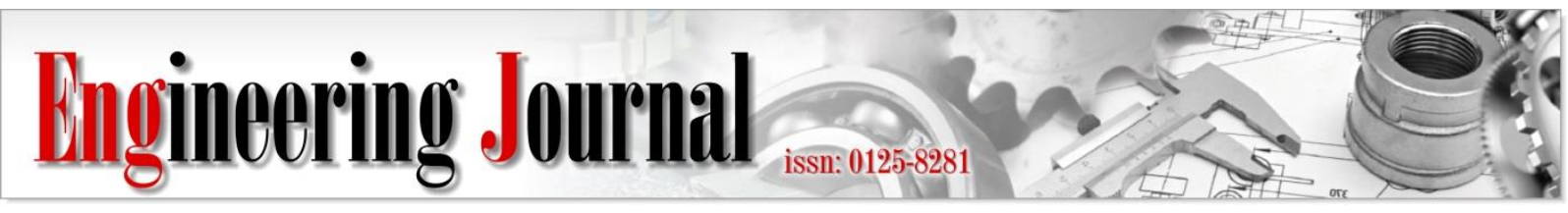

Article

\title{
Frame Size Analysis of Optimum Dynamic Tree in RFID Systems
}

\author{
Sanika K. Wjayasekara ${ }^{1}$, Muhammad Saadi ${ }^{2}$, Warakorn Srichavengsup ${ }^{3}$, Robithoh Anuur ${ }^{4}$, \\ Suvit Nakpeerayuth ${ }^{1}$, Hung-Yun $\mathrm{Hsieh}^{5}$, and Lunchakorn Wuttisittikulkij ${ }^{1, *}$ \\ 1 Smart Wireless Communication Ecosystem Research Group, Chulalongkorn University, Bangkok, \\ Thailand \\ 2 University of Central Punjab, Pakistan \\ 3 Thai-Nichi Institute of Technology, Thailand \\ 4 Tunku Abdul Rahman University, Malaysia \\ 5 National Taiwan University, Taipei,Taiwan \\ *E-mail: wlunchak@chula.ac.th (Corresponding author)
}

\begin{abstract}
In RFID (Radio Frequency Identification) system, an anti-collision algorithm plays a prominent role in the tag recognition process to reduce the required time for identifying the tag and enhance the RFID system efficiency. In this paper, we present a theoretical analysis of optimal frame size assignment for the maximization of system efficiency of a tree-based anti-collision algorithm, called optimum dynamic tree (ODT) algorithm, which is used for RFID tag recognition process. Analysis indicates that the appropriate frame size for a given number of competing tags should not be set equal to the number of tags, which is usually adopted in literature. Instead of this, the frame size should be kept smaller roughly by a factor of 0.871 to maximize system efficiency. The closed-form solution for calculating system efficiency has been derived. The simulated results show excellent correlation with the theoretical one. Range for the exact appropriate frame sizes for the number of tags is from 2 to 100 . Comparison has been made for the tag-identification time of conventional binary tree and ODT algorithms according to the international standard ISO 18000-6B.
\end{abstract}

Keywords: Binary tree, collision, efficiency, frame size, slot, tags.

ENGINEERING JOURNAL Volume 24 Issue 1

Received 28 August 2019

Accepted 27 January 2020

Published 8 February 2020

Online at http://www.engj.org/

DOI:10.4186/ej.2020.24.1.239

This article is based on the presentation at The 33rd International Technical Conference on Circuits/Systems, Computers and Communications (ITCCSCC 2018) in Bangkok, Thailand, 4th - 7th July 2018. 


\section{Introduction}

Collision Resolution Algorithm (CRA) plays a vital role in resolving the collision for a multi-access communication system. In such a system, many users randomly access the shared medium without having any prior coordination among them. As a result, collision happens when two or more user's packet overlap each other. In case of collision, the packet needs to be transmitted again. CRA determine necessary actions in order to enhance the efficiency for the identification process of the collided user/packet while improving overall system efficiency $[1,2]$.

Radio frequency identification (RFID) is a multiuser communication practice, where multiple RFID tags can simultaneously transfer the messages to an RFID reader in its interrogation zone through a shared RF channel. The RFID reader is a powerful component having enough computational memory and resources. The RFID tags range from the smart active tags to the passive tags. Active tags use their own battery power to activate while the passive tags totally depend on its reader transmission power. The passive tags are commonly deployed in RFID communication systems due to the lower cost. The main objective of an RFID reader is to identify the tags within its coverage area while utilizing lesser energy and time consumption. However, the concurrent messaging from several tags to the reader leads to a problem termed as tag collision, where the reader denies the identification of the tags. In this scenario, messages are retransmitted and consume more system resources leading to an inefficient system performance [3-6].

The CRAs are required in order to handle the tags collision problem effectively and improving the efficiency of RFID systems. There are many anti-collision algorithms for fast tag identification and can be mainly categorized into two groups namely, ALOHA and tree-based algorithms [7,8]. In an ALOHA-based algorithm such as slotted ALOHA, Framed Slotted ALOHA (FSA) and Dynamic Frame Slotted ALOHA (DFSA), the time is divided into timeslots and a set of timeslots form a frame. The tags can randomly send their responses at each slot or frame. In Frame Slotted ALOHA (FSA), a fixed frame size is being used throughout the whole process of tag identification and the maximum achievable stable efficiency is $1 / e$. In contrast, the Dynamic Framed Slotted ALOHA (DFSA) implement variable frame size depending on the number of tags in the system. Although the ALOHA-based algorithms are simple, but how many timeslots are needed for the reader to identify all tags is unknown. The tree-based algorithms sub-group the collided tags recursively in a tree structure until each leaf node of the tree structure left with zero or one tag. Hence, all tags can be known within a certain timeslot. The treebased algorithms are practically more ideal and focused in this paper [9-14].

In $[15,16]$, the tree algorithm has been used as a multiple access protocol for wireless communication systems In [16], the author has introduced a Binary Tree
Algorithm (BTA) where the collided tags are grouped into two subgroups along with the tree structure until the leaf nodes in the tree structure contain only one tag or none. BTA has an efficiency of $34.6 \%$ and can be improved up to $36.6 \%$ with the help of ternary tree algorithm (TTA), where the collided tags are resolved by further grouping them into three sub-groups. M-ary tree concept can also be found in the literature [17], where the collided tags are divided into $M$ number of sub-groups instead of two or three as in BTA or TTA respectively $[17,18]$. In addition, [16] initiated a binary tree based Optimum Dynamic Tree (ODT) algorithm with an optimum efficiency of $42.9 \%$ under infinite tag population which proved to be more efficient as compared to the conventional tree algorithms. The ODT process is performed in two steps. Initially, the tags compete in a frame where the frame size is dependent on the number of tags in the system. Secondly, the collided slots in this frame are cleared using BTA. Later in 2013, the ODT algorithm adopted in [19], and introduced a Splitting Binary Tree Slotted ALOHA (BTSA) algorithm with a system efficiency of $42 \%$. This algorithm gives the highest practical efficiency of $42 \%$ up-to-date. Therefore, it is interesting to investigate the ODT algorithm, which nearly enhances $7 \%$ of system efficiency using the splitting-based technique.

In this work, we analyzed the ODT algorithm and deduced a closed-form mathematical expression of the ODT system efficiency. Furthermore, we presented that higher system efficiency can be achieved when the frame size $(L)$ is equal to $\beta N$, where the $\beta$ is the multiplicative factor use in ODT algorithm for any range of tags $(N)$ to achieve the optimum $L$. In order to use ODT algorithm for practical RFID applications, it's easy to use lookup table which is derived for a smaller number of tags.

The rest of the paper is structured as follows. We describe the BTA and ODT algorithms in Section 2 and in Section 3, the collision arbitration process of the ISO 18000-6 TypeB protocol is given. The analysis of the ODT algorithm is shown in Section 4 with the relationship between the optimum frame sizes and the number of tags. Section 5 describes the results and discussions. We conclude our work in Section 6.

\section{The Description of Binary Tree and Optimum Dynamic Tree Algorithms}

\subsection{Basic Binary Tree Algorithm (BTA)}

Basic Binary Tree Algorithm (BTA), also known as fair tree, is originally introduced by Capetanakis [16]. In BTA, the collided users are grouped into two subgroups until the collision-free subgroups are created. In Fig. 1, a BTA execution example is presented based on the Depth First Search (DFS) considering a finite population with six users (ABCDEF) in a system.

ISO 18000-6 Type B, as the RFID air interface communication standards at $860-960 \mathrm{MHz}$ [20] uses the BTA as the collision arbitration algorithm and it has 
adopted the DFS approach in BTA in order to identify the RFID tags. According to the DFS tree branching procedure, the initially collided users (in the initial slot) can be split into two subgroups. The collided tags in the first subgroup can be further subdivided and transmitted in the respective slot. This mechanism will repeat until all the collided users in the first subgroup succeed, and then, followed by the second group of the initial slot until all the collided users succeed.

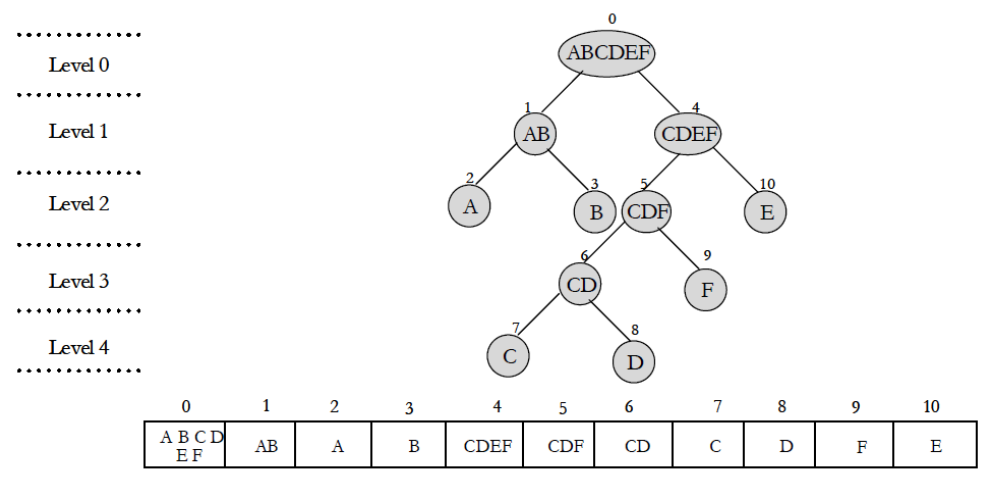

Fig. 1. An execution example of BTA with six initial collided users.

\subsection{Optimum Dynamic Tree}

The initial prototype of the optimum dynamic tree (ODT) algorithm is introduced in [16] in order to improve the average efficiency of the elemental binary tree algorithm. In the ODT algorithm, the users are initially split into K slots, without making all the users compete for the first slot which may result in a collision and a waste of a slot. Then, the collision resolution follows the same instructions of the binary tree algorithm as earlier explained. The term dynamic is used since $\mathrm{K}$ may vary according to the number of users in the system. Figure 2 shows the six tags (ABCDEF) identification processes which uses the ODT algorithm as the tag identification algorithm. In this example, we consider the initial splitting frame size as six.

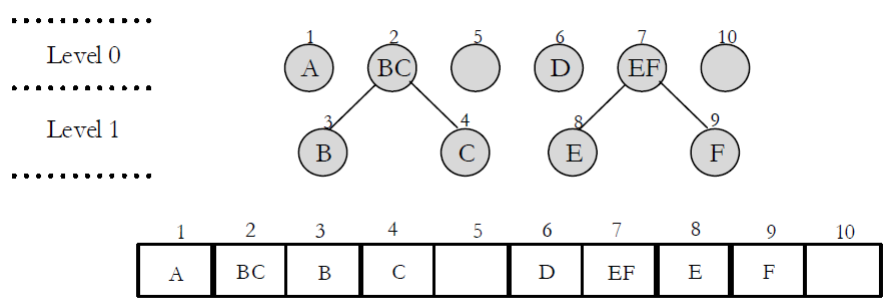

Fig. 2. An execution example of ODT for six users with initial splitting frame size of six.

\section{Binary Tree Collision Arbitration in the ISO 18000-6 Type B standard}

As aforementioned in Section 2.1, BTA is the collision arbitration algorithm used in ISO 18000-6 Type B RFID air interface communication standards at $860-960 \mathrm{MHz}$ [20]. In the Type B standard, tags maintain two types of hardware parts; a random generator to generate ' 0 ' and ' 1 ' and 8 bits counter namely COUNT. The collided tags are split into two subgroups based on the randomly generated binary value of ' 0 ' and ' 1 ' and this process is continued until all the tags are identified without any collision. The COUNT value is used to track the tag's position in the tree structure whereas the tags with the COUNT value equal to ' 0 ' can transmit their IDs to the reader.
We can summarize the collision arbitration process of Type B standard as follows in detail with the help of the tag state transition diagram shown in Fig. 3.

Step 1: The reader sends the SELECT command which is used to identify the subgroup of tags joining the collision arbitration process. The selected tags are moved from READY state to ID state and set their COUNT to ' 0 '. The tags whose COUNT value is ' 0 ' can transmit their IDs to the reader.

Step 2: If the reader receives several responses then a FAIL command is sent back to the tags.

Step 2a: The tags with COUNT ' 0 ' generate a random number ' 0 ' or ' 1 ' and add it to its counter and the rest of the tags increment the COUNT. The tags COUNT with ' 0 ' transmit their IDs again 
and other tags with none zero counter values remain silent.

Step 3: If the reader doesn't receive any response, then a SUCCESS command is sent back to the tags.

Step 3a: All the tags receiving the SUCCESS command decrement their COUNT values. Then the

tags with COUNT ' $O$ ' shall transmit their IDs to the reader.

Step 4: If the reader receives only one response then a DATA_READ command with the received ID are sent back to the corresponding tag.

Step 4a: If the corresponding tag successfully receives the DATA_READ command, it will move to the DATA EXCHANGE state and transmits its data. Then the reader sends the SUCCESS command and after receiving the SUCCESS, all the tags in the ID state decrement their counter values.

The reader maintains a counter value which is used to identify the termination of the tag identification process. When the reader's counter value becomes zero, the identification process is terminated. When the reader sends the SUCCESS command, the counter value increments by one and after sending a FAIL command, the counter value decrements by one. To terminate the tag identification process, the reader's counter value is used. Figure 4 shows an example of identifying three tags $\left(\mathrm{n}_{\mathrm{A}}\right.$, $\mathrm{n}_{\mathrm{B}}, \mathrm{n}_{\mathrm{C}}$ ) using Type B standard by following the abovementioned collision arbitration process from Step 1 to Step 4 a.

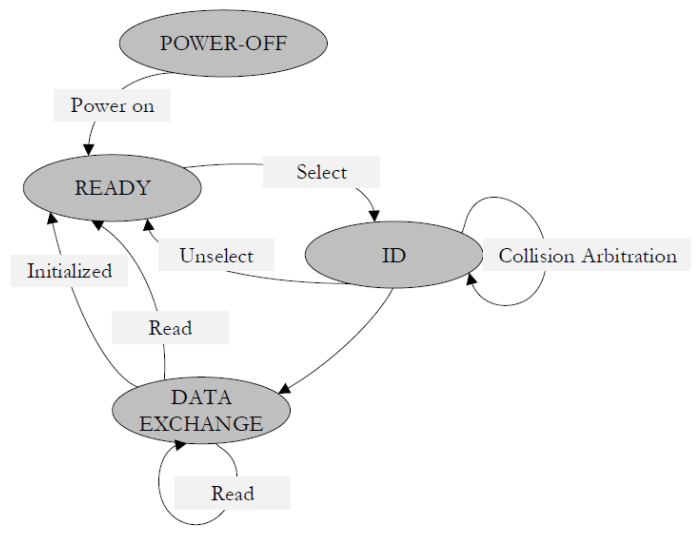

Fig. 3. State transition diagram of a tag based on ISO 18000-6 Type B standard [20].

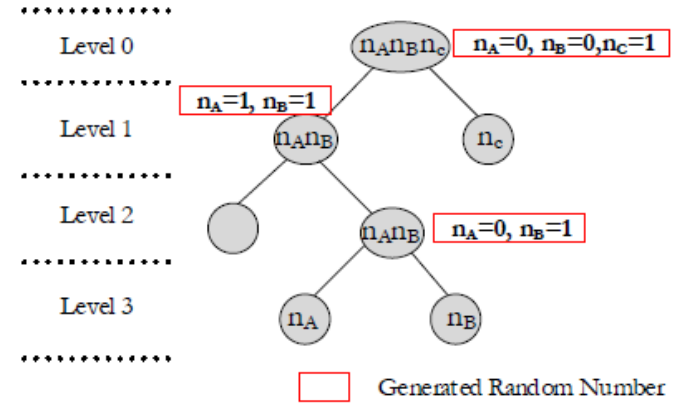

\begin{tabular}{|c|c|c|c|c|}
\hline Reader Command & \multicolumn{3}{|c|}{ Tag COUNT } & \multirow{2}{*}{ Status } \\
\cline { 2 - 4 } (Reader COUNT) & $\mathrm{n}_{\mathrm{A}}$ & $\mathrm{n}_{\mathrm{B}}$ & $\mathrm{n}_{\mathrm{C}}$ & \\
\hline SELECT(0) & 0 & 0 & 0 & Collision \\
\hline FAIL(1) & 0 & 0 & 1 & Collision \\
\hline FAIL(2) & 1 & 1 & 2 & Idle \\
\hline SUOCESS(1) & 0 & 0 & 1 & Collision \\
\hline FAIL(2) & 0 & 1 & 2 & Identified \\
\hline SUOCESS(1) & - & 0 & 1 & Identified \\
\hline SUOCESS(0) & - & - & 0 & Identified \\
\hline
\end{tabular}

Fig. 4. An example of identifying three tags using ISO 18000-6 Type B standard. 


\section{Performance Analysis of BTA and ODT Algorithms}

In this section, we present an analytical model to achieve the optimum achievable system efficiency $\eta$ of ODT algorithm. Using Eq. (1) the system efficiency is derived for $N$ tags with the mean number of timeslots required in ODT algorithm to recognize $N$ tags which is $T_{O D T}(N)$.

$$
\eta=\frac{N}{T_{O D T}(N)} .
$$

As given in [7], the mean number of timeslots desired by the binary tree algorithm with a fair splitting to recognize $N$ tags which is $T_{\text {BTA }}(N)$ can be given by

$$
T_{B T A}(N)=2\left[1+\sum_{k=1}^{\infty} C(N, k)\right]
$$

where $C(N, k)=\sum_{n=2}^{N}\left(\begin{array}{l}N \\ n\end{array}\right)\left(\frac{1}{2^{k}}\right)^{n}\left(1-\frac{1}{2^{k}}\right)^{N-n} ; C(N, k)$ represents the mean number of collision slots occur at the $k^{\text {th }}$ level of binary tree structure with fair splitting probability of $\frac{1}{2^{k}}$.

Using an approximated asymptotic expression given in [21], Eq. (2) can be further simplified and presented as Eq. (3). It shows that to identify initially collided two tags $(N=2)$ using BTA, five average number of timeslots are required including the initially collided slot. For more than two tags, it is $\frac{2 N}{\ln (2)}-1$.

$$
T_{\text {BTA }}=\left\{\begin{array}{cc}
5 & , N=2 \\
\frac{2 N}{\ln (2)}-1 & , N>2
\end{array}\right.
$$

Let $T_{O D T}(N, L)$ denotes the mean number of slots required by ODT algorithm to identify $N$ tags which are initialy distributed in a frame with $L$ slots. Then, $T_{O D T}(N, L)$ can be given by:

$$
T_{O D T}(N, L)=L \times\left[\begin{array}{r}
B(N, 1 / L, 0)+B(N, 1 / L, 1)+5 \times B(N, 1 / L, 2) \\
+\sum_{n=3}^{\infty} B(N, 1 / L, n) \times T_{B T A}(N)
\end{array}\right] .(4)
$$

where $B(n, p, i)=\left(\begin{array}{l}n \\ i\end{array}\right) p^{i}(1-p)^{n-i}$.

In ODT tag recognition process, at the earlier stage, the tags are split into $L$ sets and the collided slots among these sets are resolved based on binary tree protocol. $B(N, 1 / L, n)$ represents the binomial probability of $n$ collided tags out of $N$ tags distribute among $L$ initial slots. The $B(N, 1 / L, 0), B(N, 1 / L, 1)$ and $B(N, 1 / L, 2)$ represent the binomial probability of no tag, one tag and two tags arbitrary select $L$ slots respectively. As given in Eq. (3), the average number of slots needed to resolve two tag collision in binary tree is 5 , and $\mathrm{B}(\mathrm{N}, 1 / \mathrm{L}, 2) \times 5$ represents number of slots required to resolve two tags collision out of $N$ tags. $B(N, 1 / L, 0)+B(N, 1 / L, 1)$ represents the required number of slots for one tag selection or zero out of $N$ tags.

By substituting Eq. (3) to Eq. (4), the $T_{O D T}(N, L)$ can be simplified as given in Eq. (5).

$$
\begin{aligned}
T_{O D T}(N, L)= & \frac{2 N}{\ln (2)}-L+2\left(1-\frac{1}{L}\right)^{N-2} \\
& {\left[\frac{N(N-1)}{2 L}\left(3-\frac{2}{\ln (2)}\right)+L\left(1-\frac{1}{L}\right)^{2}+N\left(1-\frac{1}{\ln (2)}\right)\left(1-\frac{1}{L}\right)\right] }
\end{aligned}
$$

In order to discover the required optimum initial frame size in ODT to resolve the collision of any $N$ tags with minimum slot consumption, the function $T_{O D T}(N, L)$ given in Eq. (5) is differentiated with respect to $L$. Let's assume the resulting expression derived after the differentiation equal to zero and further analyze it with $L=\beta N$, where $\beta$ is a constant which represents the ratio of $L$ and $N$. Then, for large number of tags, Eq. (5) can be simplified to:

$$
e^{-1 / \beta}\left[\left(3-\frac{2}{\ln (2)}\right)\left(\frac{1}{\beta^{-1}}-1\right) \frac{1}{\beta^{2}}+2\left(\frac{1}{\beta}+1\right)+\left(1-\frac{1}{\ln (2)}\right) \frac{2}{\beta^{2}}\right]=1 .
$$

Equation (6) can be plotted as shown in Fig. 5, which illustrates the optimum frame sizes $L$, resulting into the minimum average number of timeslots in collision resolution for different number of tags. By taking the ratio between the frame size $L$ and tags, the $\beta$ value which represent the minimum average number of timeslots, can be calculated. It shows that for large number of tags, the relationship between the frame size $L$ and number of tags $N$ is $L=0.871 N$ where $\beta \approx 0.871$.

Substituting $L=0.871 \mathrm{~N}$ in Eq. (4), Eq. (7) can be derived.

$$
T_{O D T}(N, L)=2.3278 N+0.04172 \text {. }
$$

Using Eq. (1), the system efficiency of ODT algorithm for large number of tags is

$$
\eta_{O D T} \approx \frac{N}{2.3278 N} \approx 0.42959 .
$$

As given in Eq. (8), the optimal system efficiency of ODT algorithm is 0.42959 . The results in this paper 
provide the confirmation for the results in reference [19] in which the authors derived the optimal efficiency of ODT with the assumption of initial frame size equal to the number of tags in the system. In our work, we obtain the optimum average efficiency of 0.429 based on the relationship between the initially required frame size and the available number of tags in the system.

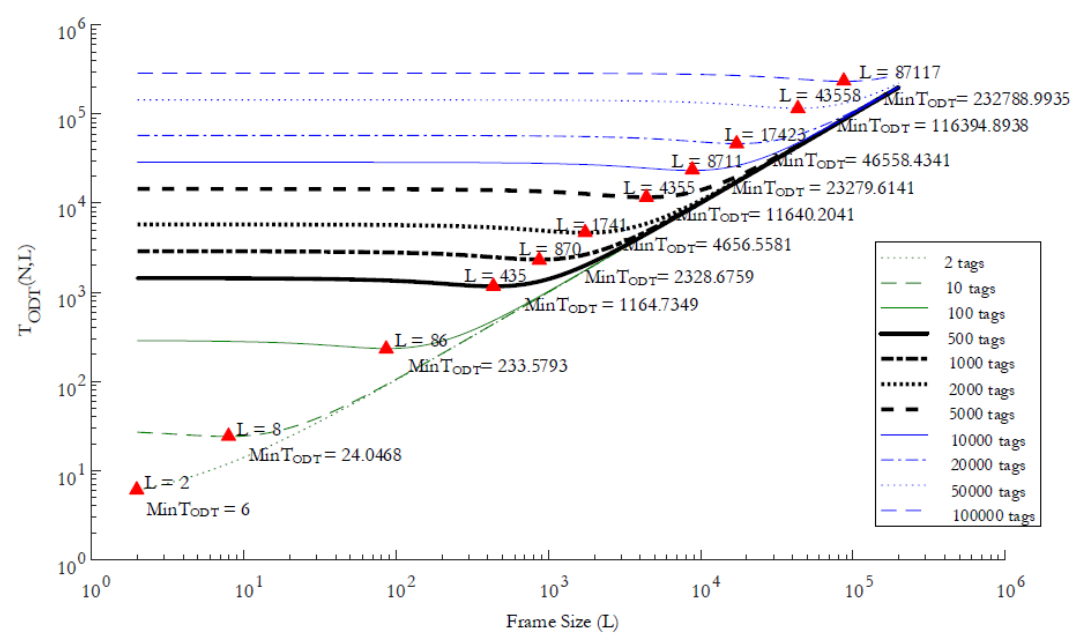

Fig. 5. Frame sizes $(L)$ vs. $T_{O D T}(N, L)$ for different number of $N$.

\section{Simulation Results and Discussion}

In this section, we validate the analytical model given in Section 4 using computer simulation for 2-1000 number of tags $(N)$. The subsequent impact on the performance of ODT algorithm with different $\beta$ values for $2-1000$ number of tags is evaluated. Furthermore, for binary tree and ODT algorithms, performances are compared using ISO/IEC 18000-6 Type B standard with respect to required average time to identify all the tags. The processes of tags selecting the slots and reader identifying the tags are performed using MATLAB simulator.

Figure 6 shows the achievable system efficiency of ODT algorithm for different number of tags and $\beta$ values. It confirms that the highest achievable efficiency is derived when $0.75 \leq \beta<1$. For small number of tags ranging from 2 to 10 , it is required to have different $\beta$ values to achieve the highest system efficiency as illustrated in Fig. 6(a). For larger number of tags as shown in Fig. 6(b) and Fig. 6(c), the optimal efficiency can be achieved with $\beta \approx 0.87$. Figure 7 illustrates that for number of tags less than 150 , there is a rapid change in the ratio of $L / N$, while for larger number of tags, it is approximated to 0.8 . Therefore, the simulated results support the analytically derived $\beta$ value in Section 4 . As we can see in Fig. 6(a), for $2 \leq N \leq 100$ the $\beta$ value is inconsistent. Therefore, in order to use ODT algorithm in practical RFID applications, a lookup table can be easily derived as given Table 1 for $2 \leq N \leq 100$ range of tags. For larger number of tags such as $N>100$, the frame sizes can be computed using $\operatorname{round}(0.871 \times N)$ where $\operatorname{round}(\cdot)$ is used to round the digits to nearest integer.

We analyzed the ODT efficiency for three different $\beta$ values as given in Fig. 8 . The optimal $\beta$ means that $\beta$ give the optimum efficiency in ODT algorithm for $N$. $\beta=0.871$ indicates the ODT efficiency when the initial frame size $L$ equal to $0.871 \mathrm{~N}$ as given in Section 4 . $\beta=1$ represents that the initial frame size $L$ is equal to number of tags $N$. The curve of $\beta=0.871$ is vibrated close to the curve of optimal $\beta$ confirming that the $\beta=0.871$ gives the ODT optimum efficiency around $42.9 \%$ for large $N$ as given in (8). Furthermore, it is justified that the frame size should be lower than the number of tags to achieve the higher system efficiency. 


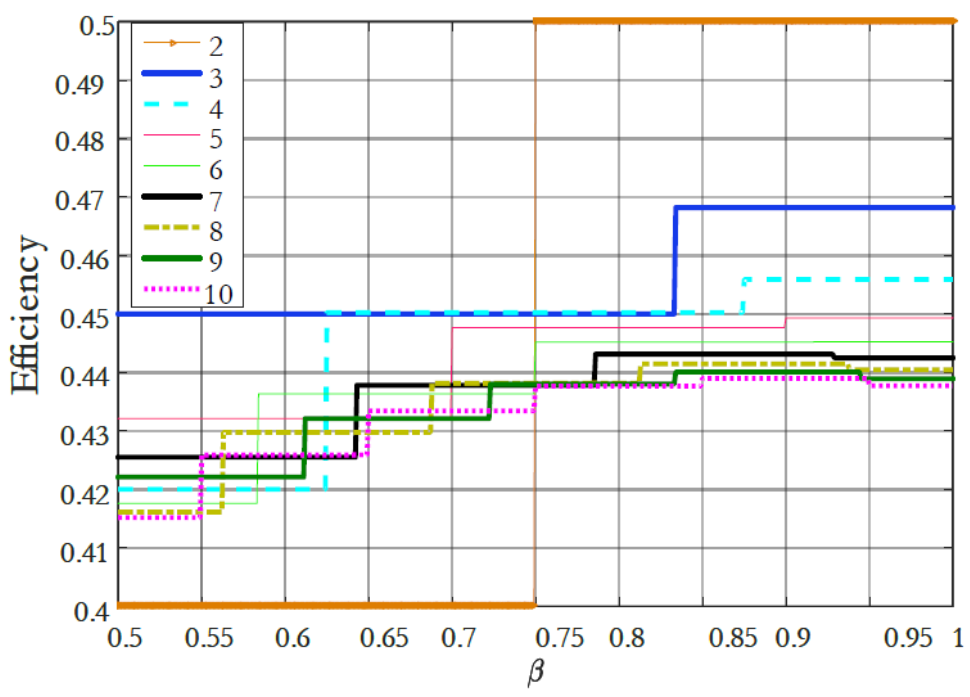

(a)

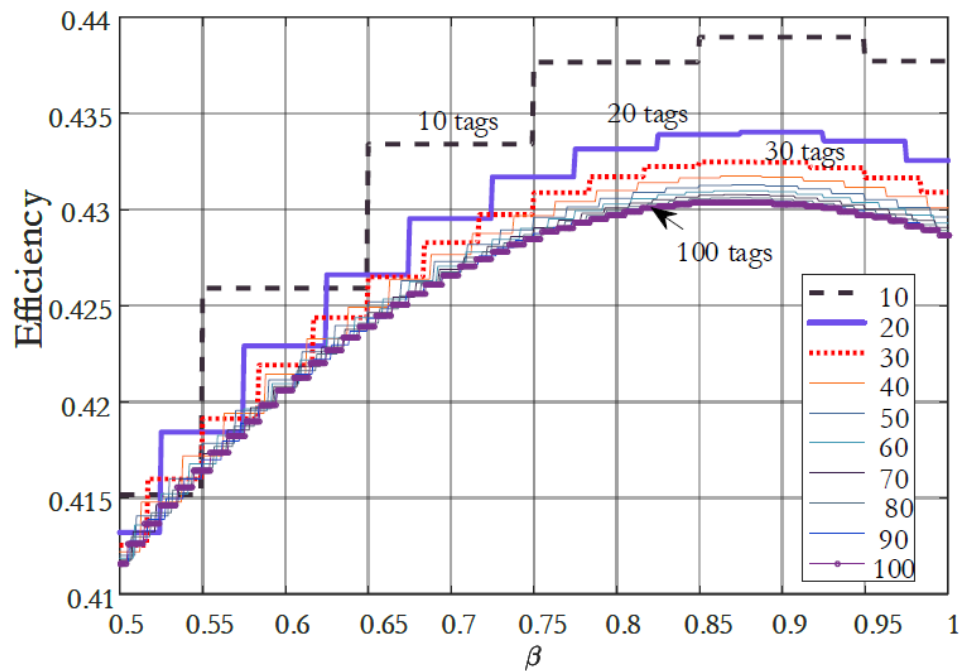

(b)

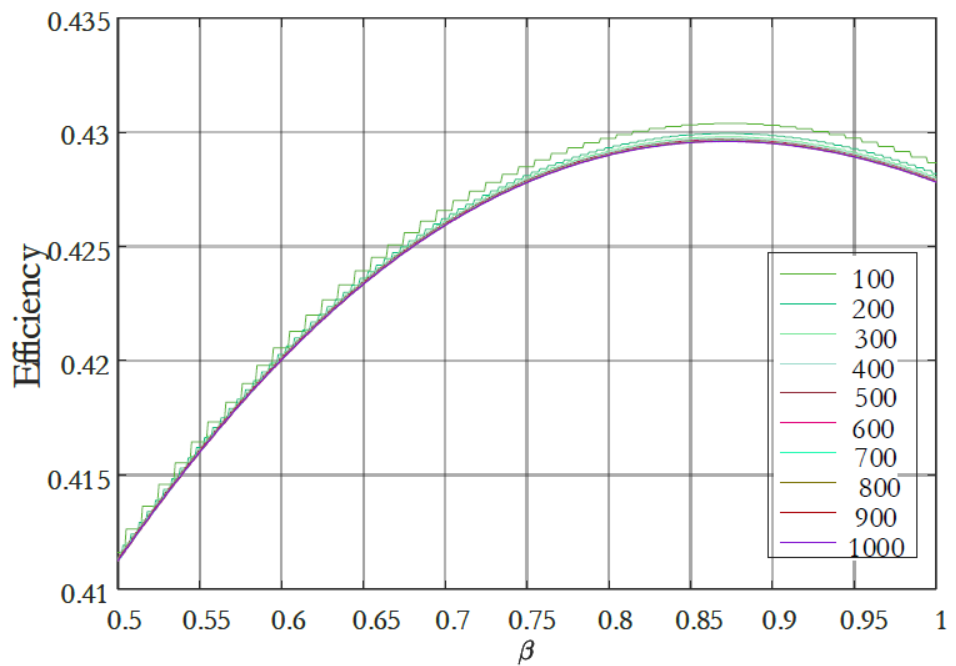

(c)

Fig. 6. ODT algorithm efficiency for different $\beta$. 


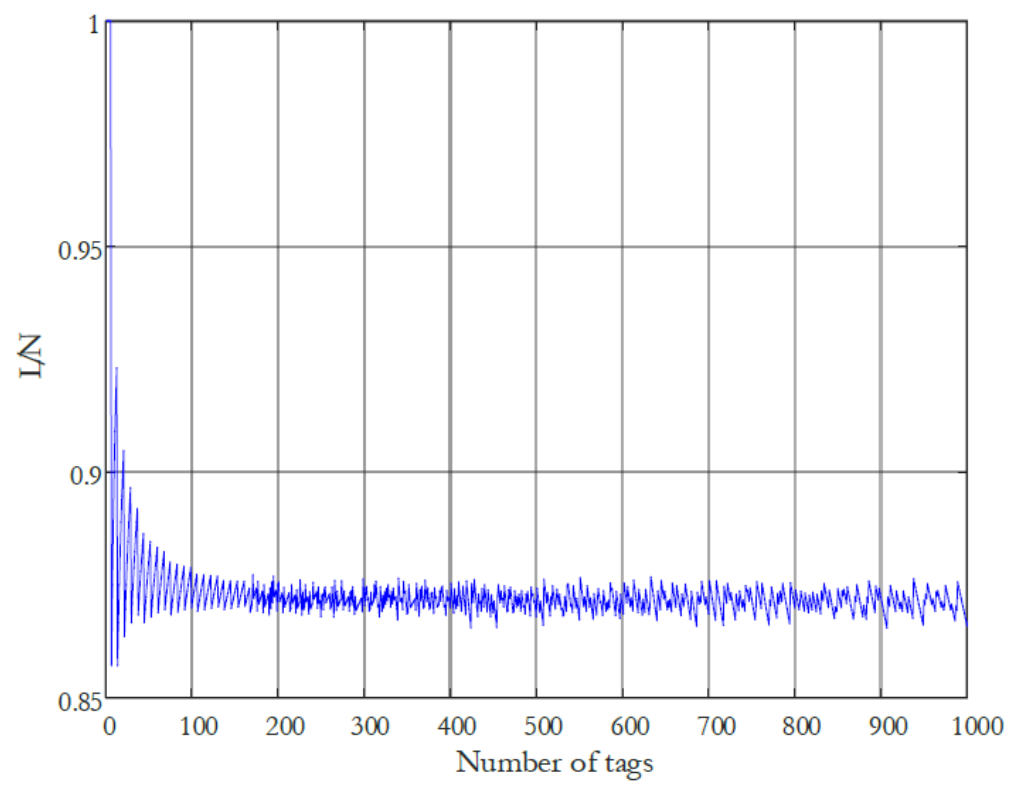

Fig. 7. $L / N$ for number of tags $2 \leq N \leq 1000$.

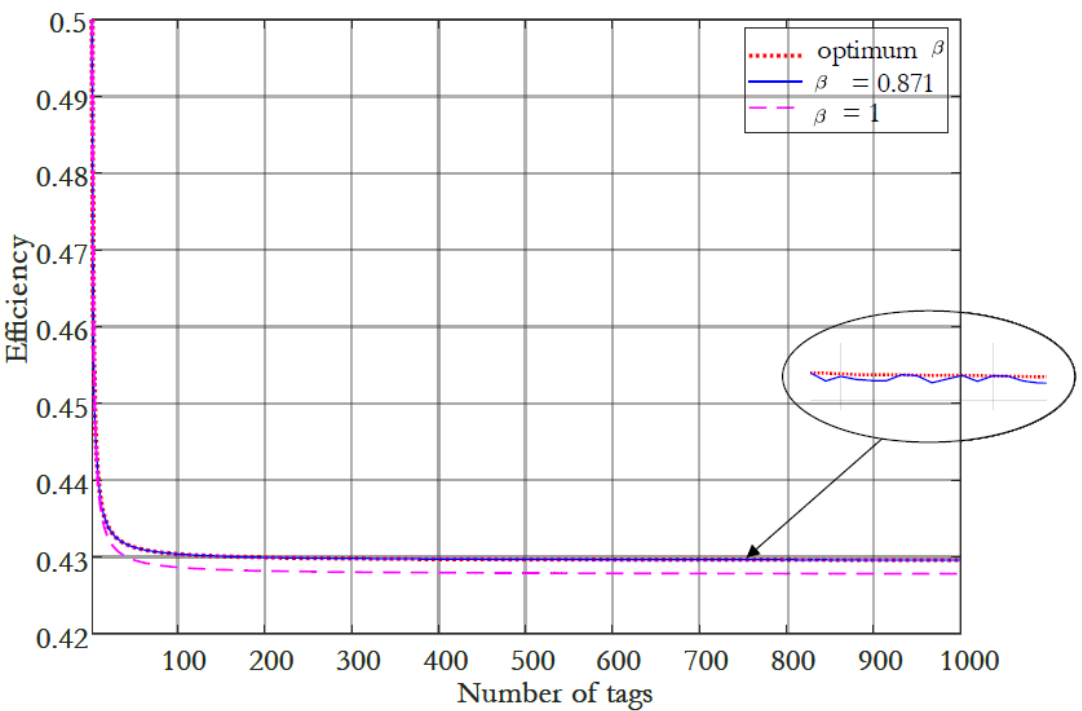

Fig. 8. ODT algorithm efficiency for number of tags $2 \leq N \leq 1000$.

Table 1. Lookup table with optimal frame sizes for number of tags $1 \leq N \leq 100$.

\begin{tabular}{|l|l|l|l|l|l|l|l|l|l|l|l|l|l|l|l|l|l|l|l|l|}
\hline $\boldsymbol{N}$ & 1 & 2 & 3 & 4 & 5 & 6 & 7 & 8 & 9 & 10 & 11 & 12 & 13 & 14 & 15 & 16 & 17 & 18 & 19 & 20 \\
\hline $\boldsymbol{L}$ & 1 & 2 & 3 & 4 & 5 & 6 & 6 & 7 & 8 & 9 & 10 & 11 & 12 & 12 & 13 & 14 & 15 & 16 & 17 & 18 \\
\hline $\boldsymbol{N}$ & 21 & 22 & 23 & 24 & 25 & 26 & 27 & 28 & 29 & 30 & 31 & 32 & 33 & 34 & 35 & 36 & 37 & 38 & 39 & 40 \\
\hline $\boldsymbol{L}$ & 19 & 19 & 20 & 21 & 22 & 23 & 24 & 25 & 26 & 26 & 27 & 28 & 29 & 30 & 31 & 32 & 33 & 33 & 34 & 35 \\
\hline $\boldsymbol{N}$ & 41 & 42 & 43 & 44 & 45 & 46 & 47 & 48 & 49 & 50 & 51 & 52 & 53 & 54 & 55 & 56 & 57 & 58 & 59 & 60 \\
\hline $\boldsymbol{L}$ & 36 & 37 & 38 & 39 & 39 & 40 & 41 & 42 & 43 & 44 & 45 & 46 & 46 & 47 & 48 & 49 & 50 & 51 & 52 & 53 \\
\hline $\boldsymbol{N}$ & 61 & 62 & 63 & 64 & 65 & 66 & 67 & 68 & 69 & 70 & 71 & 72 & 73 & 74 & 75 & 76 & 77 & 78 & 79 & 80 \\
\hline $\boldsymbol{L}$ & 53 & 54 & 55 & 56 & 57 & 58 & 59 & 60 & 60 & 61 & 62 & 63 & 64 & 65 & 66 & 66 & 67 & 68 & 69 & 70 \\
\hline $\boldsymbol{N}$ & 81 & 82 & 83 & 84 & 85 & 86 & 87 & 88 & 89 & 90 & 91 & 92 & 93 & 94 & 95 & 96 & 97 & 98 & 99 & 100 \\
\hline $\boldsymbol{L}$ & 71 & 72 & 73 & 73 & 74 & 75 & 76 & 77 & 78 & 79 & 80 & 80 & 81 & 82 & 83 & 84 & 85 & 86 & 87 & 87 \\
\hline
\end{tabular}


Additionally, the performance of BTA and ODT algorithms in terms of the tag-identification time in seconds is being evaluated using ISO/IEC 18000-6 Type $\mathrm{B}$ standard as given in [22]. We consider the data rate between reader and tag is $40 \mathrm{kbps}$ and reader wait for 16 bits of Quite field for tags response. Tags wait for reader

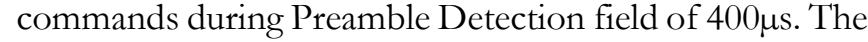
timing values given in Table 2 are being utilize where the tag identification time is calculated based on the number of reader commands and tag responses in bits and multiply it with the data rates of both reader to tag and tag to reader [20]. As depicted in Fig. 9, the ODT algorithm has a higher identification speed than the BTA algorithm. However, the given results for ODT algorithm assume the perfect condition in which the number of tags in the system is known. Therefore, the depicted ODT algorithm results in Fig. 9 are satisfied only with a good estimator.

Table 2 Time for processing one node based on Type B standard [22].

\begin{tabular}{|c|c|c|c|}
\hline & Collision & One tag reply & Idle \\
\hline $\begin{array}{c}\text { Time for processing } \\
\text { one node (ms) }\end{array}$ & 4.025 & 9.975 & 2.025 \\
\hline
\end{tabular}

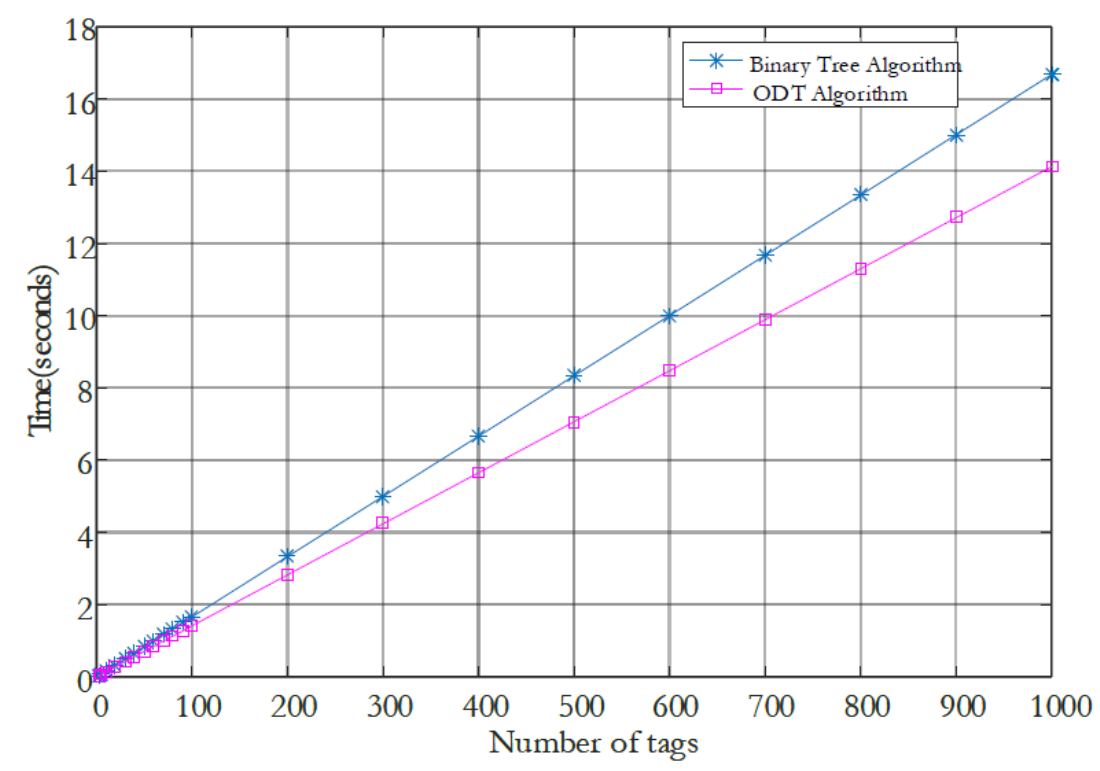

Fig. 9. Average identification for different number of tags $2 \leq N \leq 1000$ using Type B standard.

\section{Conclusion}

In this paper, we theoretically analyzed the relationship between the frame size and the number of tags for the ODT algorithm. The theoretical values are in good agreement with the simulation results. The results confirm that to achieve the optimum efficiency in the ODT algorithm, the initial frame size should be less than the number of tags. Furthermore, the multiplicative factor of $\beta$ is identified for different ranges of tags and for a small number of tags, it shows a rapid change while for a large number of tags, it becomes constant around 0.871. Therefore, for a small number of tags, we have introduced a lookup table with optimum frame sizes to be used in practical RFID applications. Further, it confirms that the ODT algorithm can apply to the type B RFID systems with a good tag estimator for fast tag identification.

\section{Acknowledgement}

We thank Ratchadapisek Somphot Fund for Postdoctoral Fellowship, Chulalongkorn University for the research support.

\section{References}

[1] P. H. Cole, L. H. Turner, Z. Hu, and D. C. Ranasinghe, "The next generation of RFID technology," in Unique Radio Innovation for the 21st Century: Building Scalable and Global RFID Networks, D. C. Ranasinghe, Q. Z. Sheng, and S. Zeadally, Eds. Berlin, Heidelberg: Springer Berlin Heidelberg, 2011, pp. 3-23.

[2] J. L. Massey, "Collision-resolution algorithms and random-access communications," in Multi-User Communication Systems. Springer, 1981, pp. 73-137.

[3] S. Nakpeerayuth, L. Wuttisittikulkij, P. Vanichchanunt, W. Srichavengsup, N. Wattanamongkhol, R. Annur, M. Saadi, K. 
Wannakong, and S. Siwamogsatham, "Efficient medium access control protocols for broadband wireless communications," in Advanced Trends in Wireless Communications. InTech, 2011, p. 353.

[4] D. K. Klair, K. W. Chin, and R. Raad, "A survey and tutorial of RFID anti-collision protocols," IEEE Communications Surveys \& Tutorials, vol. 12, no. 3, pp. 400-421.

[5] Z. Tang and Y. He, "Research of multi-access and anti-collision protocols in RFID systems," in International Workshop on Anti-Counterfeiting, Security and Identification (ASID), Xiamen, Fujian, 2007 pp. $377-$ 380, 2007.

[6] D.-H. Shih, P.-L. Sun, D. C. Yen, and S.-M. Huang, "Taxonomy and survey of RFID anti-collision protocols," Computer Communications, vol. 29, no. 11, pp. 2150-2166, 2006.

[7] S. K. Wijayasekara, S. Nakpeerayuth, R. Annur, W. Srichavengsup, K. Sandrasegaran, H. Y. Hsieh, and L. Wuttisittikulkij, "A collision resolution algorithm for RFID using modified dynamic tree with Bayesian tag estimation," IEEE Communications Letters, vol. 22, no. 11, pp. 2238-2241, Nov. 2018.

[8] S. Wijayasekara, A. Robithoh, P. Sasithong, P. Vanichchanunt, S. Nakpeerayuth, and L. Wuttisittikulkij, "A reduced complexity of Vahedi's tag estimation method for DFSA," Engineering Journal, vol. 21, no. 6, pp. 111-125, Oct. 2017.

[9] N. Abramson, "THE ALOHA SYSTEM: Another alternative for computer communications," in The Proceedings of the November 17-19, 1970, Fall Joint Computer Conference, Houston, Texas, 1970, pp. 281285.

[10] L. G. Roberts, "ALOHA packet system with and without slots and capture," Computer Communications Review, vol. 5, no. 2, pp. 28-42, April 1975.

[11] F. Schoute, "Dynamic frame length ALOHA," IEEE Transactions on Communications, vol. 31, no. 4, pp. 565-568, 1983.

[12] J. Su, Z. Sheng, D. Hong, and G. Wen, “An effective frame breaking policy for dynamic framed slotted Aloha in RFID," IEEE Communications Letters, vol. 20, no. 4, pp. 692-695, 2016.
[13] Y. Chen, J. Su, and W. Yi, “An efficient and easy-toimplement tag identification algorithm for UHF RFID systems," IEEE Communications Letters, vol. 21, no. 7, pp. 1509-1512, July 2017.

[14] J. Su, Z. Sheng, and L. Xie, "A collision-tolerantbased anti-collision algorithm for large scale RFID system," vol. 21, no. 7, pp. 1517-1520, July 2017.

[15] J. Capetanakis, "Tree algorithms for packet broadcast channels," IEEE Transactions on Information Theory, vol. 25, no. 5, pp. 505-515, 1979.

[16] J. Capetanakis, "Generalized TDMA: The multiaccessing tree protocol," IEEE Transactions on Communications, vol. 27, no. 10, pp. 1476-1484, 1979.

[17] P. Mathys and P. Flajolet, "Q-ary collision resolution algorithms in random-access systems with free or blocked channel access," IEEE Transactions on Information Theory, vol. 31, no. 2, pp. 217-243, 1985.

[18] Y. C. Lai, L. Y. Hsiao, and B. S. Lin, "Optimal slot assignment for binary tracking tree protocol in RFID tag identification," IEEE/ACM Transactions on Networking, vol. 23, no. 1, pp. 255-268, Feb. 2015.

[19] H. Wu, Y. Zeng, J. Feng, and Y. Gu, "Binary tree slotted ALOHA for passive RFID tag anticollision," IEEE Transactions on Parallel and Distributed Systems, vol. 24, no. 1, pp. 19-31, 2013.

[20] Information Technology Radio-frequency Identification for Item Management-Part 6: Parameters for Air Interface Communications at $860 \mathrm{MHz}$ to $960 \mathrm{MHz}$, ISO-IEC 18000-6, 2004

[21] F. Borgonovo and M. Cesana, "ARPA: An arbitration protocol based on advanced channel feedback for radio frequency identification," in 2008 IEEE International Conference on Wireless and Mobile Computing, Networking and Communications, 2008, pp. 178-183.

[22] D. K. Kwon, W.-J. Kim, and H.-N. Kim, "Improvement of Anti-Collision Performance for the ISO 18000-6 Type B RFID System," IEICE Transactions on Communications, vol. 90, no. 8, pp. 21202125, 2007.

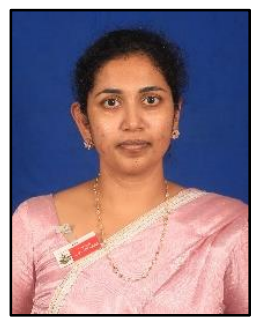

Sanika K. Wijyasekara received her B.Sc. degree in Information Technology specialized in Computer Systems and Networking from Sri Lanka Institute of Information Technology, Sri Lanka, in 2010 and the M.Sc. degree from the Asian Institute of Technology, Thailand, in 2012. She has obtained her Ph.D. degree from Department of Electrical Engineering, Chulalongkorn University, Bangkok, Thailand, in 2019. During 2018 to 2019 she was a research visiting student with the Mobile Networks and Wireless Communications (TONIC) Research Group at National Taiwan University, Taiwan. 


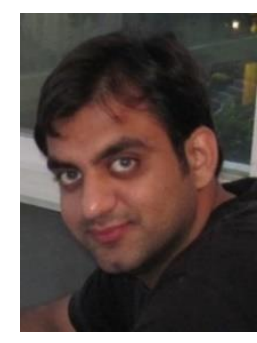

Muhammad Saadi is currently working as an Assistant Professor at Department of Electrical Engineering, Faculty of Engineering, University of Central Punjab. He received his Ph.D from Department of Electrical Engineering, Chulalongkorn University, Thailand. During his Ph.D., he was associated with NECTEC and KMUTNB as a research assistant and lecturer respectively. Furthermore, he also worked as technical content writer at Aimagin. Mr. Saadi has completed his Bachelor's Degree from National University of Computer and Emerging Sciences, Pakistan in year 2007. Before pursuing his Master's Degree from National University of Malaysia (UKM) in year 2008, he has worked as Network Engineer in Mobilink, Pakistan.

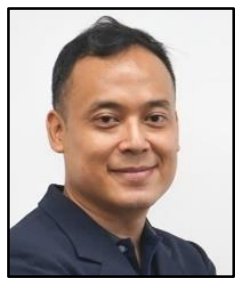

Warakorn Srichavengsup obtained the B.Eng., M.Eng. and Ph.D. degree in electrical engineering from Chulalongkorn University, Bangkok, Thailand, in 1998, 2003 and 2009, respectively. He is currently a lecturer with the Department of Computer Engineering at Thai-Nichi Institute of Technology (TNI), Bangkok, Thailand. Prior to joining TNI, he was a visiting research student during 2008 with the Laboratory for Information and Decision Systems (LIDS) at the Massachusetts Institute of Technology (MIT). His main research interests are MAC protocol for high speed wireless local area networks.

Robithoh Anuur, photograph and biography not available at the time of publication.

Suvit Nakpeerayuth, photograph and biography not available at the time of publication.

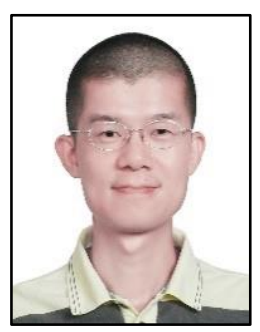

Hung-Yun Hsieh received his Ph.D. degree in Electrical and Computer Engineering from Georgia Institute of Technology, Atlanta, GA, USA. He is currently a Professor at the Department of Electrical Engineering and Graduate Institute of Communication Engineering in National Taiwan University, Taiwan. His research interest includes wireless communications, mobile computing, and network science.

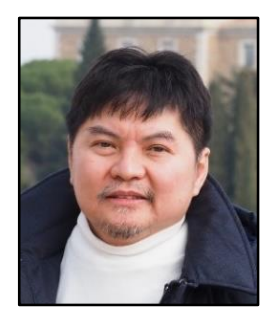

Lunchakorn Wuttisittikulkij was born in Bangkok, Thailand. He received the B.Eng degree in Electrical Engienering, Chulalongkorn University, Thailand, M.Sc. degree in Telecommunication and Information System from the University of Essex, UK and the Ph.D. degree in Electronic System Engineering from the University of Essex, UK. Since 1997, he joined Chulalongkorn University as a lecturer. He is the co-author of 15 books and more than 100 articles. His research interests include optical network design and optimization, medium access control protocols, virtual reality for education, wireless communications and $5 \mathrm{G}$ networks.. 